\title{
September 2014 Imaging Case of the Month
}

\author{
Michael B. Gotway, MD \\ Department of Radiology \\ Mayo Clinic Arizona \\ Scottsdale, AZ
}

Clinical History: A 57-year-old non-smoking woman presented to her physician as an outpatient with complaints of worsening cough, fever, chills, and shortness of breath. The patient's past medical history includes systemic lupus erythematosus diagnosed 18 years earlier, for which the patient has been variably treated with corticosteroids, hydroxychloroquine, and azathioprine. Additional past medical and surgical history includes migraines, mood disorder, diabetes mellitus treated with metformin, hysterectomy for endometriosis, and iron-deficient anemia. The patient was also diagnosed with small lymphocytic lymphoma 3 years earlier following a right breast biopsy when an abnormal opacity was discovered incidentally at routine screening breast imaging. She has not been treated for this neoplasm as no B symptoms have been reported.

Frontal and lateral chest radiography (Figure 1) was performed.

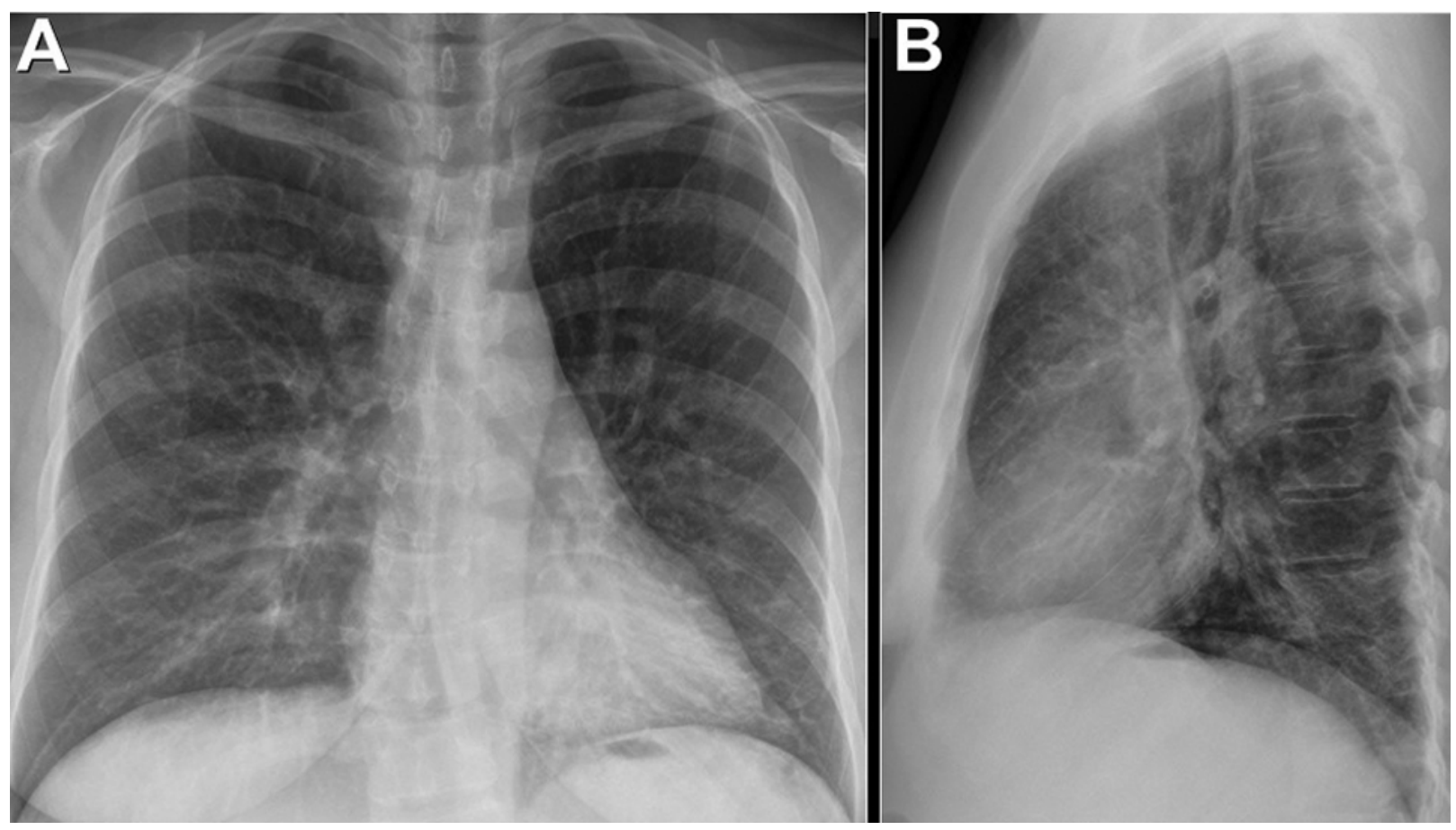

Figure 1. Panel A: Initial frontal chest x-ray. Panel B: Initial lateral chest x-ray. 
Which of the following statements regarding the chest radiograph is most accurate?

1. The chest radiograph shows asymmetric pulmonary vascularity

2. The chest radiograph shows bilateral linear and reticular opacities and diminished lung volumes suggesting fibrotic lung disease

3. The chest radiograph shows linear and curvilinear opacities suggesting cystic lung disease

4. The chest radiograph shows mild streaky central opacities, possibly reflecting airway thickening

5. The chest radiograph shows numerous small nodules 


\section{Correct! \\ 4. The chest radiograph shows mild streaky central opacities, possibly reflecting airway thickening}

The pulmonary vascularity is normal and symmetric bilaterally, and there is no evidence of small nodules in either lung. Lung volumes appear normal. There is some mild central interstitial thickening that may represent airway thickening, manifesting as streaky opacities, best seen in the retrocardiac region on the lateral projection. While some linear opacities are present, these findings are not basal predominant and are not suggestive of fibrotic lung disease. No features to suggest cystic pulmonary abnormalities, including curvilinear opacities (which can reflect visualization of the walls of cysts) are present.

The patient was treated with azithromycin, with some improvement in symptoms, although not complete resolution, and she presented to the emergency room with essentially the same complaints several days later.

Which of the following represents the next, most appropriate step for the assessment of the findings at chest radiography?

1. ${ }^{18}$ FDG-PET scan

2. Fluoroscopic-guided percutaneous transthoracic needle biopsy

3. Repeat chest radiography

4. Thoracic MRI

5. Ventilation - perfusion scintigraphy 


\section{Correct! \\ 3. Repeat chest radiography}

The clinical history suggests the possibility of an acute lower respiratory tract infection and the recent chest radiograph is non-specific, but at least not unsupportive of this working diagnosis. As the main clinical concern is pulmonary parenchymal infection, thoracic MRI plays no role in the evaluation of this patient. The role of ${ }^{18} \mathrm{FDG}-\mathrm{PET}$ scanning is largely limited to the evaluation of indeterminate solitary pulmonary nodules and staging of primary lung, pleural, and esophageal malignancies, or assessment of suspected metastatic disease in the setting of extrathoracic neoplasia, and is generally not employed in the setting of suspected acute infection. Percutaneous transthoracic needle biopsy is typically utilized to target solitary or multiple pulmonary nodules, or pleural or chest wall lesions, for tissue sampling, and no such target is available in this circumstance. Ventilation-perfusion scintigraphy is most often used to assess patients for suspected venous thromboembolism, and so could play a role in the assessment of a patient with thoracic symptoms associated with an as yet unconfirmed nonthromboembolic etiology for those symptoms, but the absence of thromboembolic risk factors and the patients presenting complaints point more towards lower respiratory tract infection rather than pulmonary embolism; therefore, repeat chest radiography is the most appropriate choice of those listed above.

Repeat chest radiography (Figure 2) was performed.

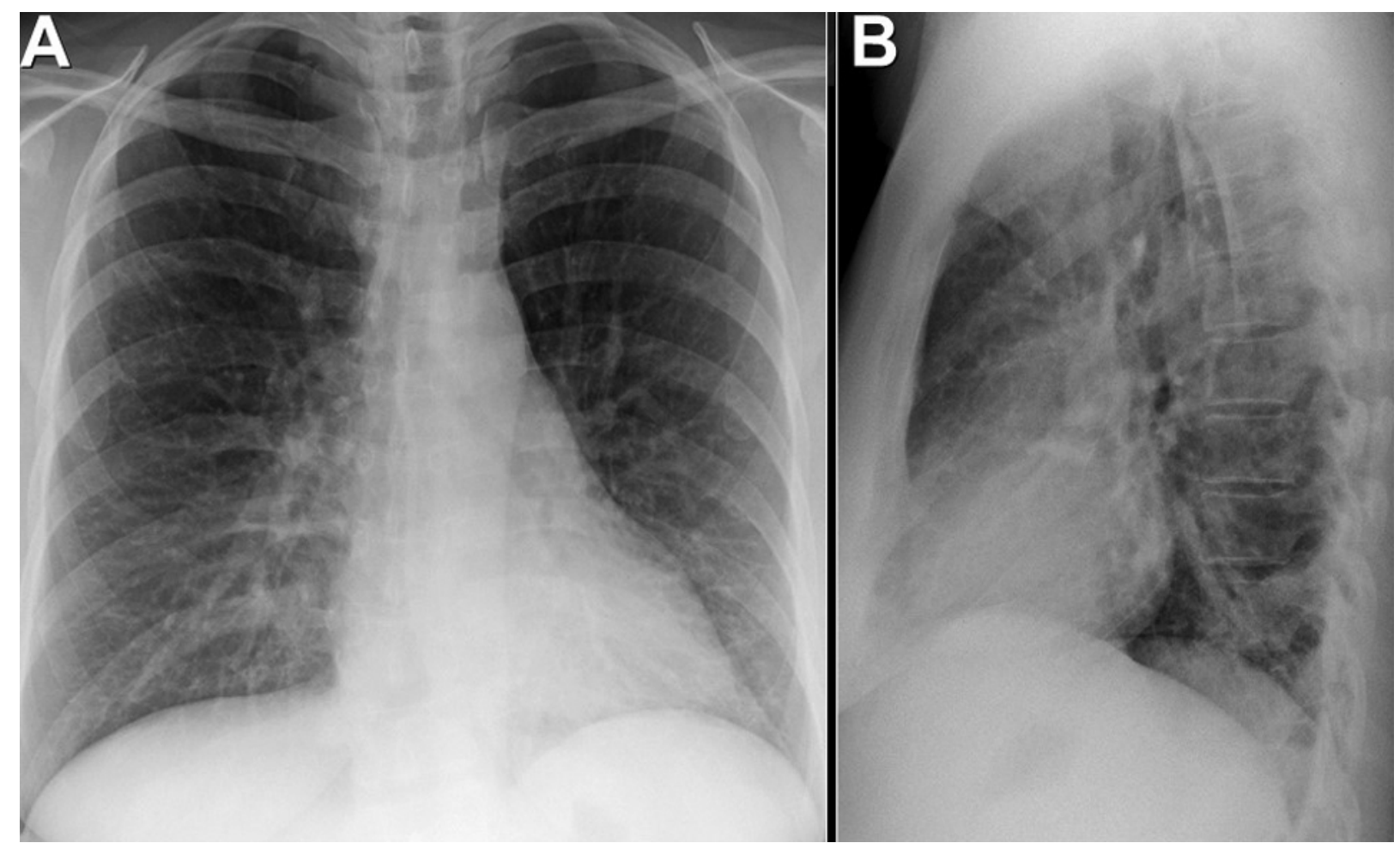

Figure 2. Panel A: Repeat frontal chest x-ray. Panel B: Repeat lateral chest x-ray. 
Which of the following statements regarding the chest radiograph is most accurate?

1. The chest radiograph shows development of consolidation suggesting pneumonia

2. The chest radiograph shows development of linear opacities and interlobular septal thickening suggesting increased pressure (hydrostatic) edema

3. The chest radiograph shows interval development of peribronchial and mediastinal lymphadenopathy

4. The chest radiograph shows multiple new thin-walled cystic lesions and cavities

5. The chest radiograph shows no significant change from prior 


\section{Correct!}

\section{The chest radiograph shows no significant change from prior}

The hilar and mediastinal contours remain normal and no new areas of pulmonary consolidation are seen. No features to suggest increased pressure (hydrostatic) edema, such as interlobular septal thickening, central perihilar haziness, or pleural effusions. No evidence of cystic or cavitary lung disease is present. The chest radiograph appears relatively unchanged compared with the presentation imaging (Figure 1).

Clinical course: The patient subsequently underwent unenhanced thoracic CT (Figure $3)$.

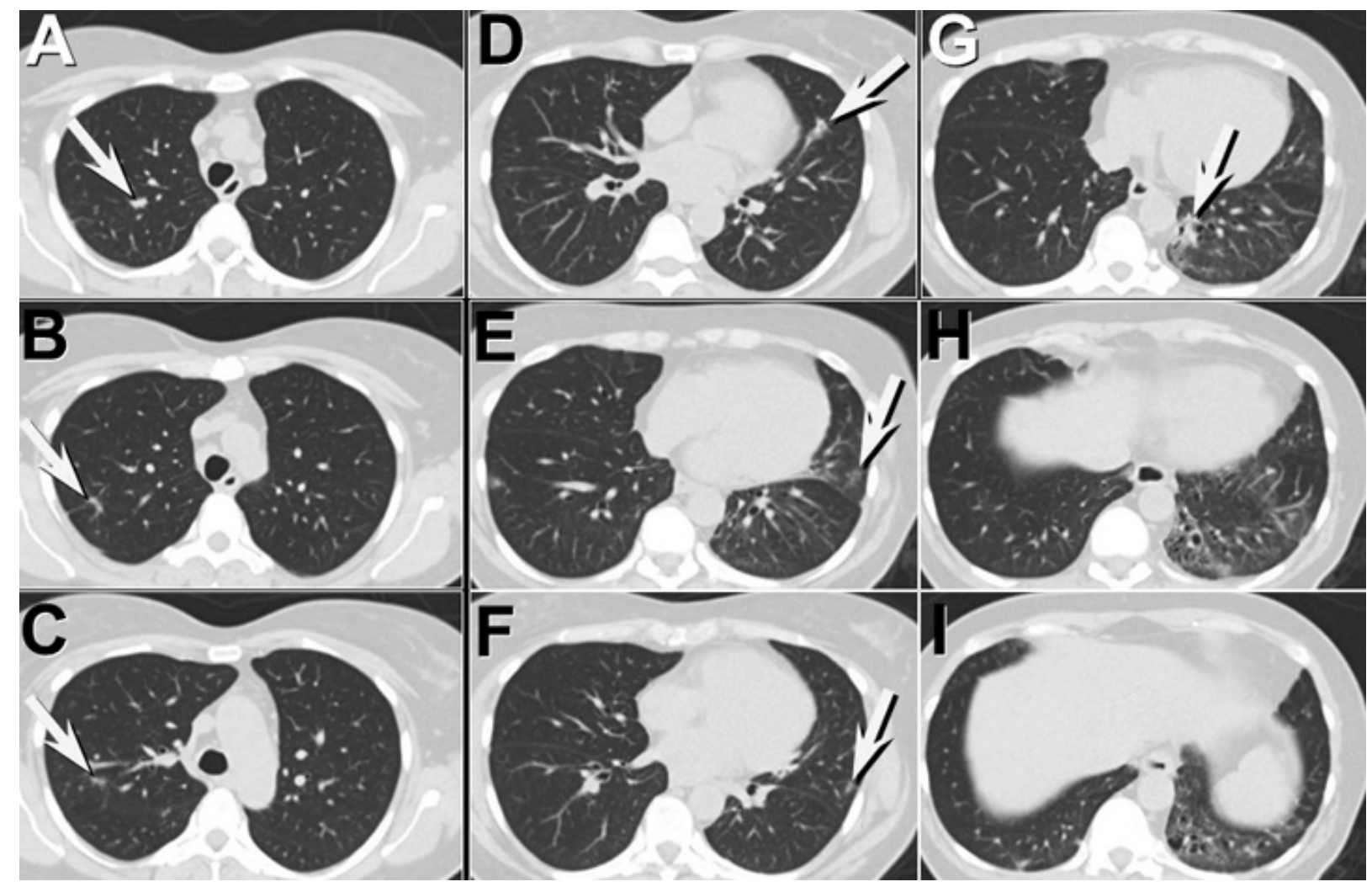

Figure 3. Thoracic CT shows multiple abnormalities (arrows, panels A-G).

Which of the following statements regarding this imaging study is most accurate?

1. The thoracic CT shows diffuse centrilobular nodules

2. The thoracic CT shows miliary nodules

3. The thoracic CT shows multiple non-specific small nodular opacities and basal peribronchial infiltration and bronchiectasis

4. The thoracic CT shows numerous thin-walled cystic lesions

5. The thoracic CT shows upper lobe predominant fibrotic changes 


\section{Correct!}

\section{The thoracic CT shows multiple non-specific small nodular opacities and basal}

peribronchial infiltration and bronchiectasis

While several nodules are present on the thoracic $\mathrm{CT}$, the nodules are relatively few in number and somewhat peribronchiolar in distribution, and therefore represent neither diffuse centrilobular nodules nor miliary nodules. Basal fibrotic changes, consisting of peribronchiolar infiltration and bronchiectasis are present, but these findings are not seen within the upper lobes. No thin-walled cystic lesions are present.

Clinical course: The patient was treated periodically for presumed lower respiratory tract infections, with occasional temporary improvement in symptoms, but her symptoms never completely resolved, nor was a causative organism ever isolated. She underwent repeat chest radiography (Figure 4) several months following her initial presentation, which showed similar findings to her two prior chest radiographs (Figures 1 and 2), perhaps with slight worsening of the basal peribronchial thickening.

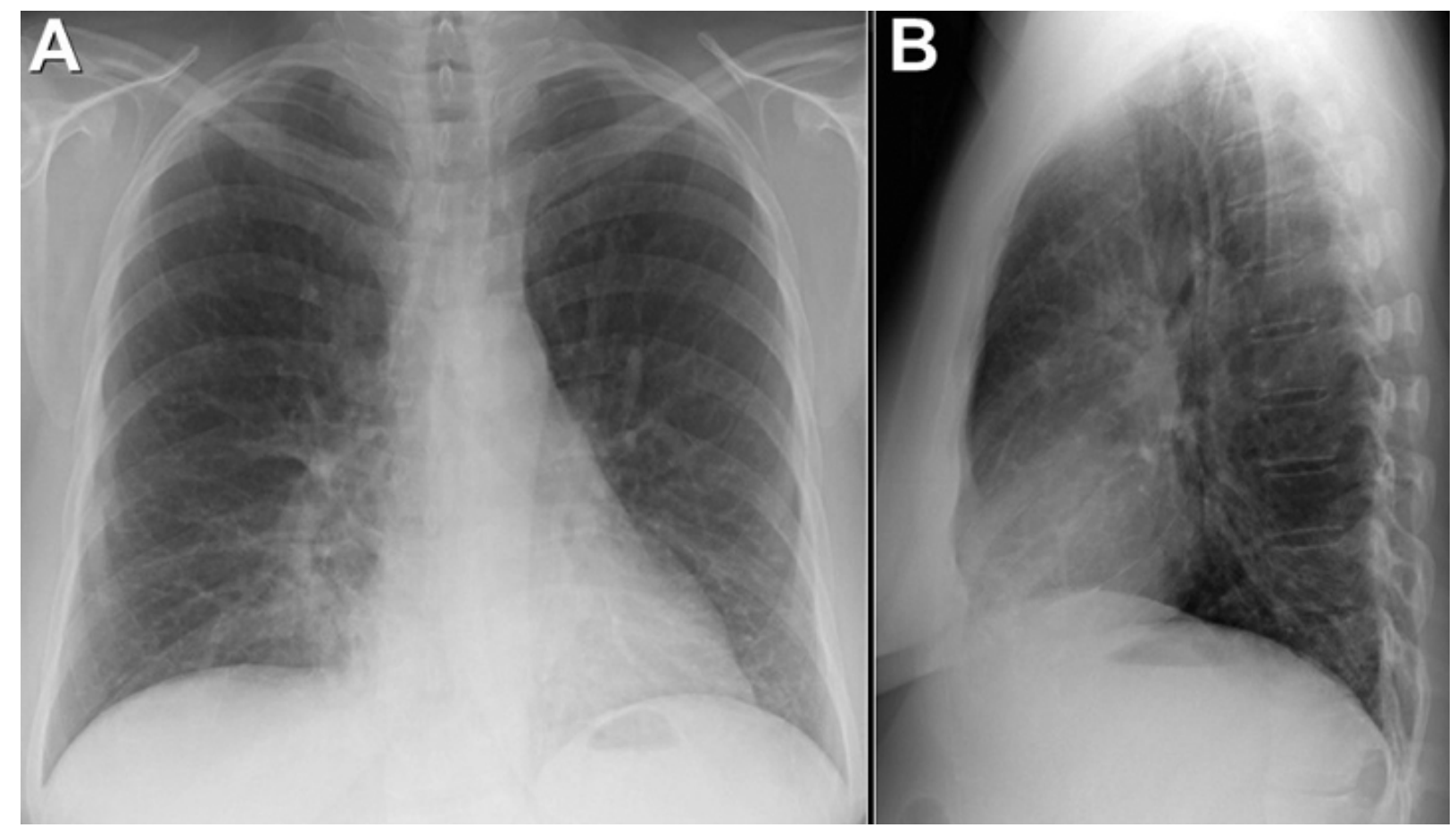

Figure 4. Repeat chest radiography several months after initial presentation.

Nearly one year following her initial presentation, she complained of persistent, now worsening, symptoms, and underwent repeat chest radiography (Figure 5). 


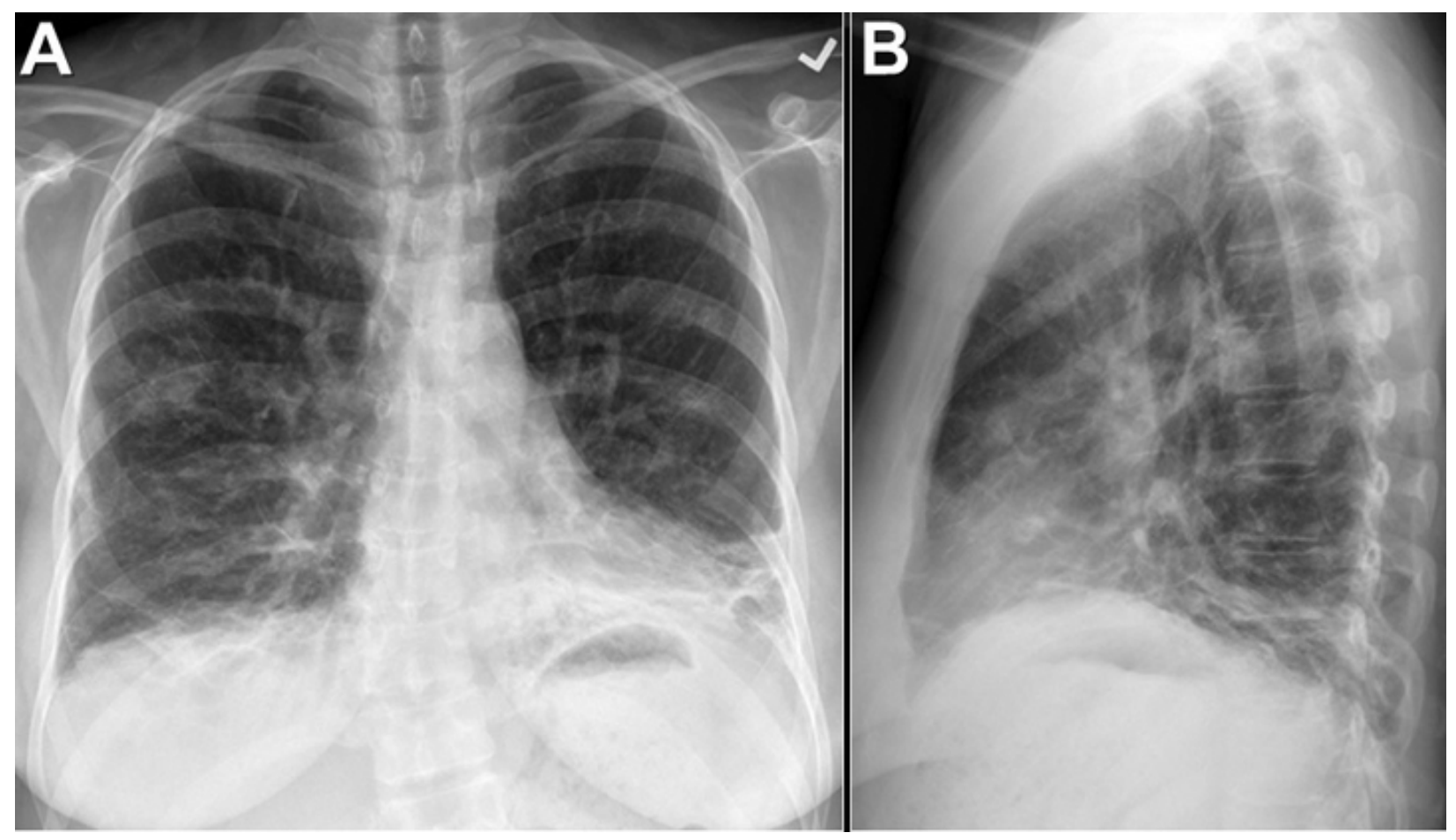

Figure 5. Repeat chest radiography taken nearly one year after initial presentation.

Which of the following statements regarding this imaging study is most accurate?

1. The chest radiograph shows improvement in the previously noted bronchovascular thickening

2. The chest radiograph shows interval development of peribronchial and mediastinal lymphadenopathy

3. The chest radiograph shows interval worsening of the findings noted previously, now with worsening basal linear opacities and more poorly defined right mid-lung opacity

4. The chest radiograph shows multiple new thin-walled cystic lesions and cavities

5. The chest radiograph shows no significant change from prior 


\section{Correct!}

3. The chest radiograph shows interval worsening of the findings noted previously, now with worsening basal linear opacities and more poorly defined right mid-lung opacity

The frontal and lateral chest radiograph shows diminished lung volumes with clear interval worsening of basal predominant interstitial thickening and peribronchial infiltration, with new right mid-lung poorly defined opacity. No cystic or cavitary lesions are present, and there is no clear evidence of either hilar / peribronchial or mediastinal lymph node enlargement.

Clinical course: The patient subsequently underwent repeat thoracic CT (Figure 6).

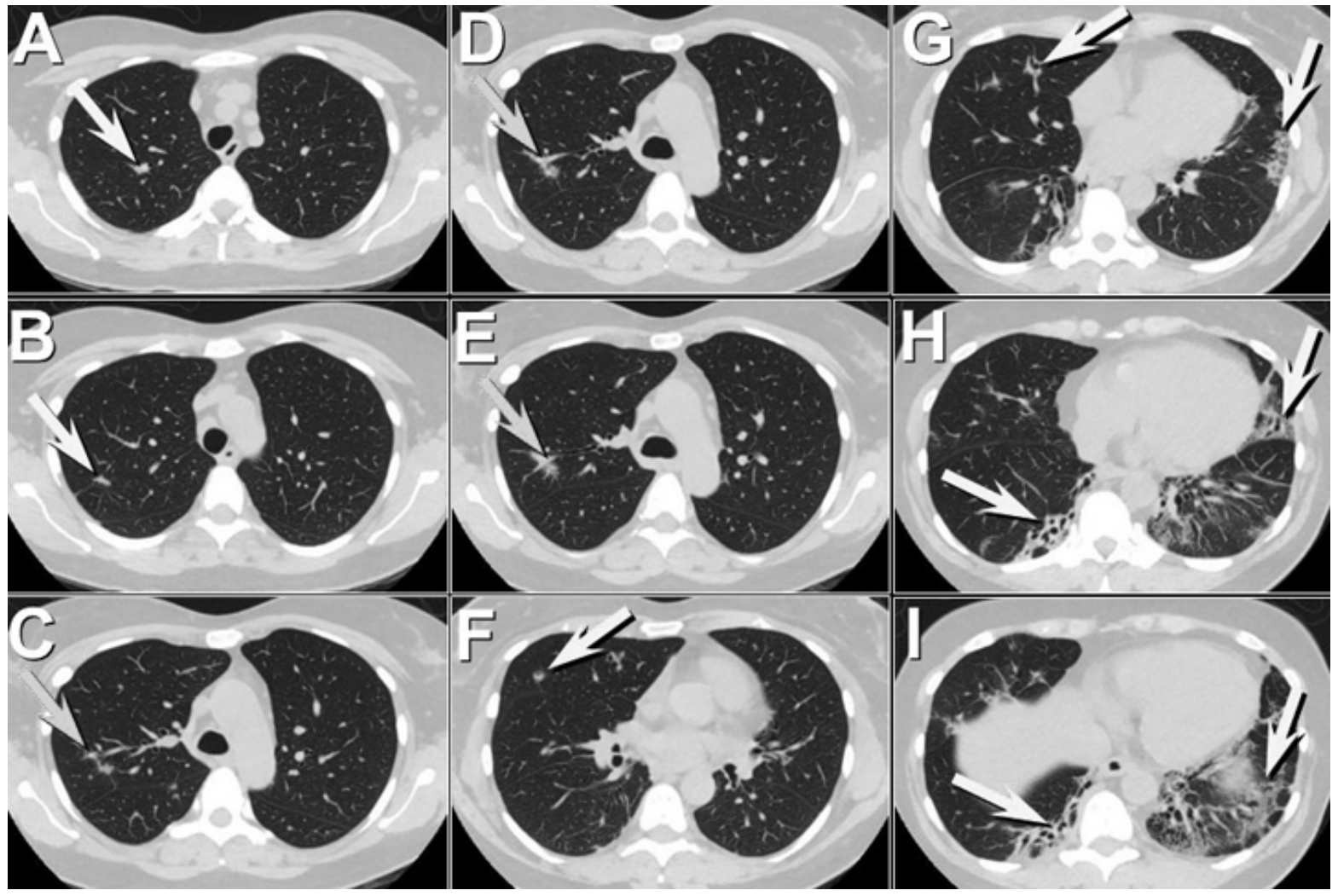

Figure 6. Thoracic CT shows multiple abnormalities (arrows, panels A-F).

Which of the following statements regarding this imaging study is most accurate?

1. The thoracic CT shows interval worsening of pre-existing nodules and bibasilar peribronchial infiltration, ground-glass opacity, and bronchiectasis

2. The thoracic CT shows new peribronchial and mediastinal lymph node enlargement

3. The thoracic CT shows new pleural effusions

4. The thoracic CT shows new upper lobe bronchiectasis

5. The thoracic CT shows regression in the previously noted nodular opacities 


\section{Correct!}

\section{The thoracic CT shows interval worsening of pre-existing nodules and bibasilar peribronchial infiltration, ground-glass opacity, and bronchiectasis}

The thoracic CT shows worsening of pre-existing nodules and bibasilar peribronchial infiltration, ground-glass opacity, and bronchiectasis. In particular, at least one nodule in the right upper lobe (see panels C-E, Figure 6) has shown enlargement. No clear evidence of upper lobe bronchiectasis, pleural effusions, or peribronchial / hilar and mediastinal lymph node enlargement is noted.

Clinical course: The patient subsequently underwent another repeat thoracic CT just over one month later (Figure 7), which suggested worsening of the basal peribronchial opacities, enlargement of the dominant right upper lobe nodule, and several new nodular opacities bilaterally.

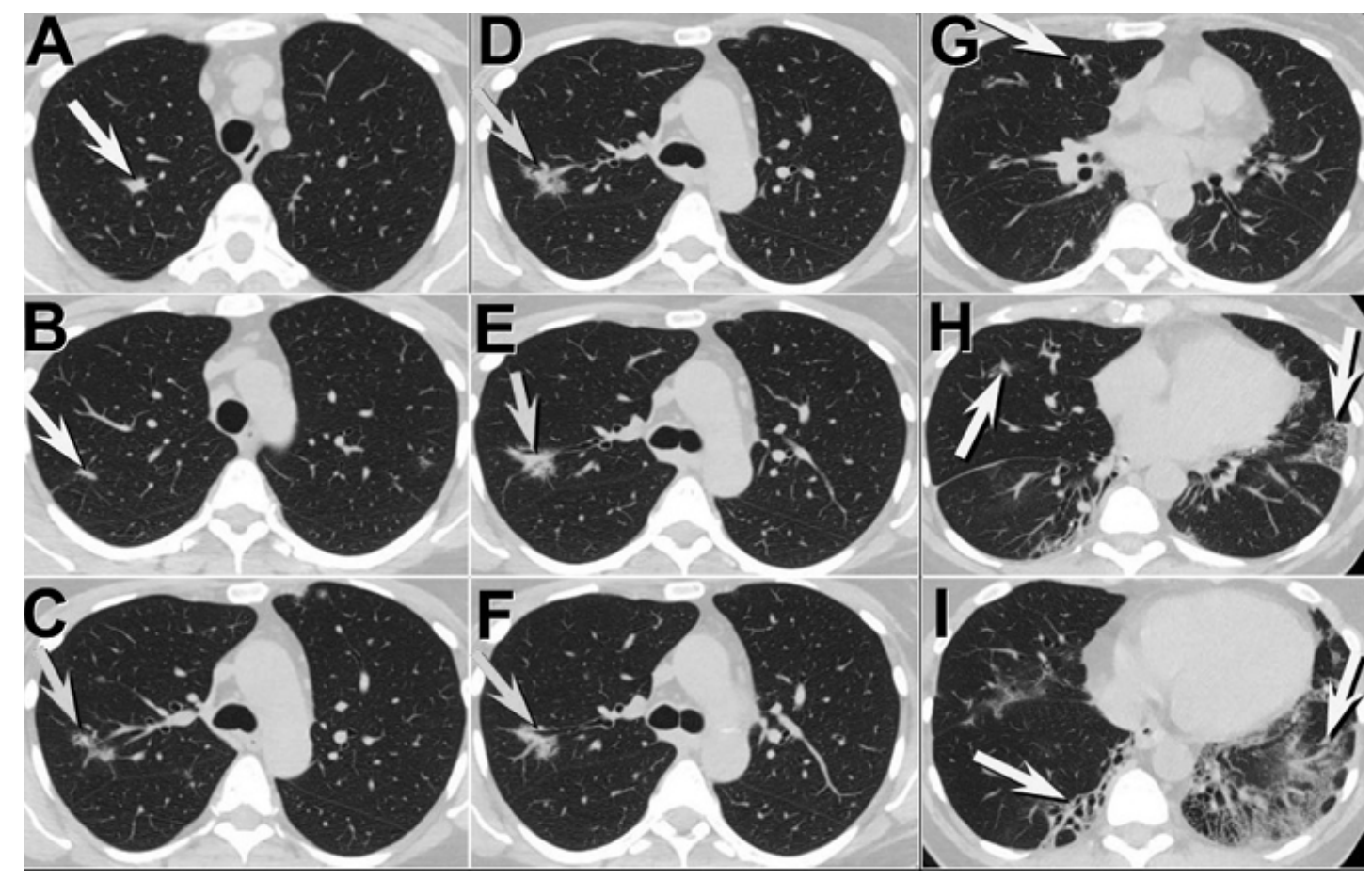

Figure 7. Repeat thoracic CT scan again showing worsening of the basal peribronchial opacities, enlargement of the dominant right upper lobe nodule, and several new nodular opacities bilaterally.

Which of the following represents the most appropriate next step for the management of this patient?

1. ${ }^{18}$ FDG-PET scan

2. ${ }^{68} \mathrm{Ga}$-citrate scanning

3. Pulmonary function testing

4. Repeat thoracic CT with intravenous contrast

5. Ventilation-perfusion scanning 


\section{Correct! \\ 1. ${ }^{18}$ FDG-PET scan}

${ }^{18}$ FDG-PET scanning is the most appropriate procedure among those listed above for the evaluation of this patient. ${ }^{18} \mathrm{FDG}-\mathrm{PET}$ scanning has the ability to assess for additional sites of hypermetabolic tissue, perhaps outside of the thorax and potentially unsuspected, that may change the differential diagnostic considerations or provide an additional target for tissue sampling. ${ }^{68} \mathrm{Ga}$-citrate scanning (aka "gallium" scan) could show uptake in the opacities, but such uptake is relatively non-specific, and may be seen with inflammatory or malignant etiologies, and negative results would not provide any useful data. Repeating the thoracic CT is unlikely to be of benefit when two recent thoracic CTs have shown progressive abnormalities; the addition of intravenous contrast is unlikely to add any management-altering information. Ventilation-perfusions scanning plays a role in the assessment of suspected thromboembolic disease and assessing lung function prior to surgery, and, rarely evaluation patients for shunts to the systemic circulation, but such disorders are not among the differential diagnostic considerations for this patient. Finally, while assessment of pulmonary function may benefit this patient, such assessment is not germane as regards determining the next step in the evaluation of this patient's worsening status.

Further clinical course: The patient subsequently underwent ${ }^{18}$ FDG-PET scanning (Figure 8), which showed evidence of variable, primarily mild-to-moderate, metabolic in many of the pulmonary opacities, but no extrathoracic sites of hypermetabolic tissue.

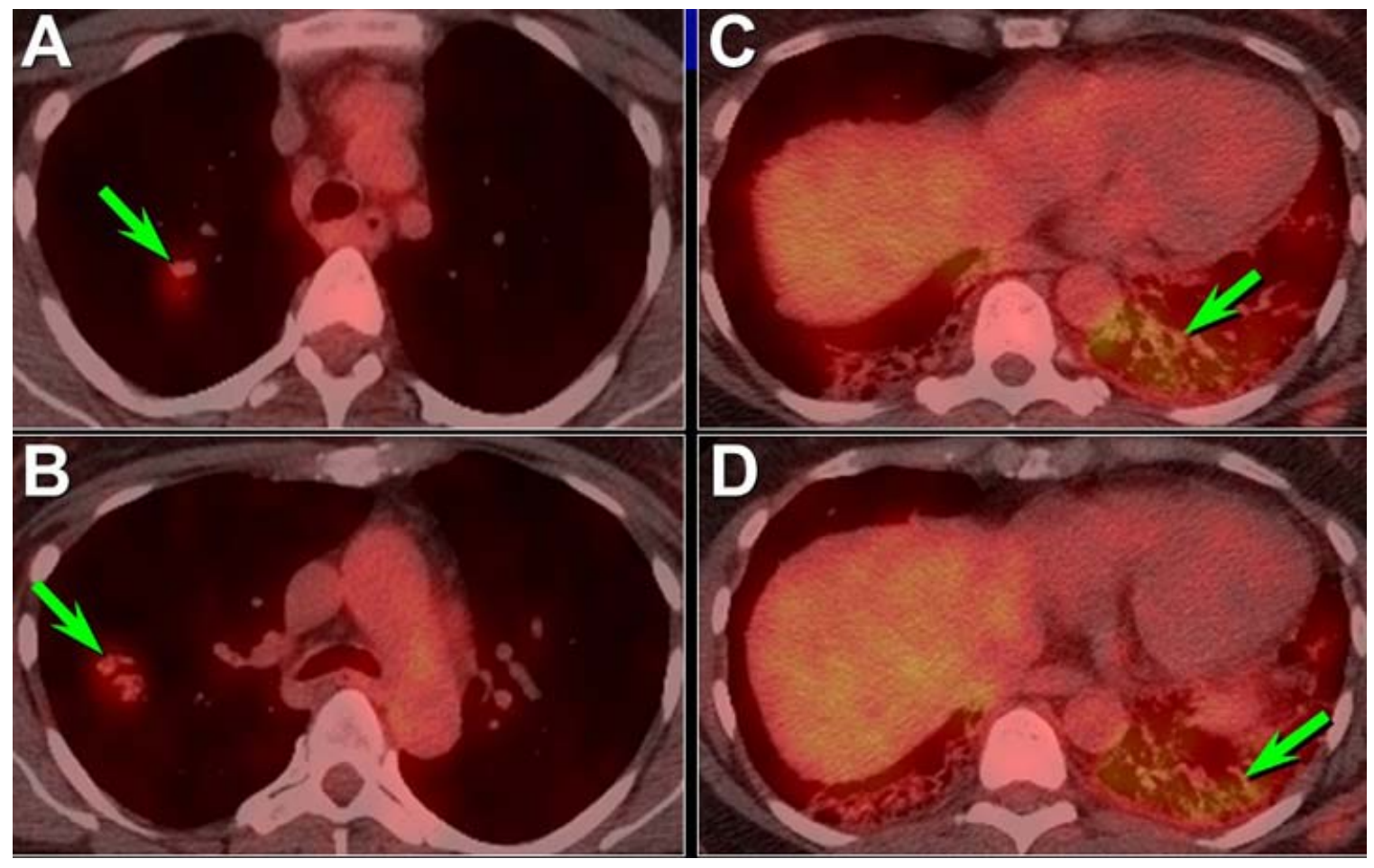

Figure $8 .{ }^{18}$ FDG-PET scanning shows mild-to-moderate metabolic activity in several of the pulmonary opacities (arrows). 
Which of the following represents the most appropriate next step for the management of this patient?

1. Bronchoscopy with bronchoalveolar lavage and transbronchial biopsy

2. Percutaneous fine needle aspiration / core biopsy

3. Thoracoscopic lung biopsy

4. All of the above

5. None of the above 


\section{Correct! \\ 4. All of the above}

The first three choices- thoracoscopic lung biopsy, bronchoscopy, and percutaneous fine needle aspiration / core biopsy- all represent methods for obtaining a tissue diagnosis, which is indicated in this patient with progressive clinical symptoms and imaging findings. Thoracoscopic lung biopsy has the potential advantage of being the procedure most likely to provide a definitive diagnosis, although it is the most invasive, potentially morbid, and expensive among those listed. On the other hand, while bronchoscopy with bronchoalveolar lavage and transbronchial biopsy may have a lesser capability for providing a definitive diagnosis, it is the last invasive; percutaneous transthoracic fine needle aspiration / core biopsy is intermediate between thoracoscopic lung biopsy and bronchoscopy as regards both diagnostic capabilities and complication rate. The choice of procedure in this circumstance largely rests with available expertise and patient preference.

Further clinical course: The patient subsequently underwent thoracoscopic lung biopsy, which showed bronchiectasis, chronic bronchitis, bronchiolectasis, and chronic bronchiolitis with prominent peribronchiolar metaplasia, mucostasis, organizing pneumonia, and lymphoid hyperplasia. Tissue eosinophilia was present, but no features to suggest allergic bronchopulmonary aspergillosis were seen. The sample also showed marked lymphoid hyperplasia but no convincing evidence of malignant lymphoma was present. The patient subsequently underwent repeat thoracic CT (Figure 9) several months later.

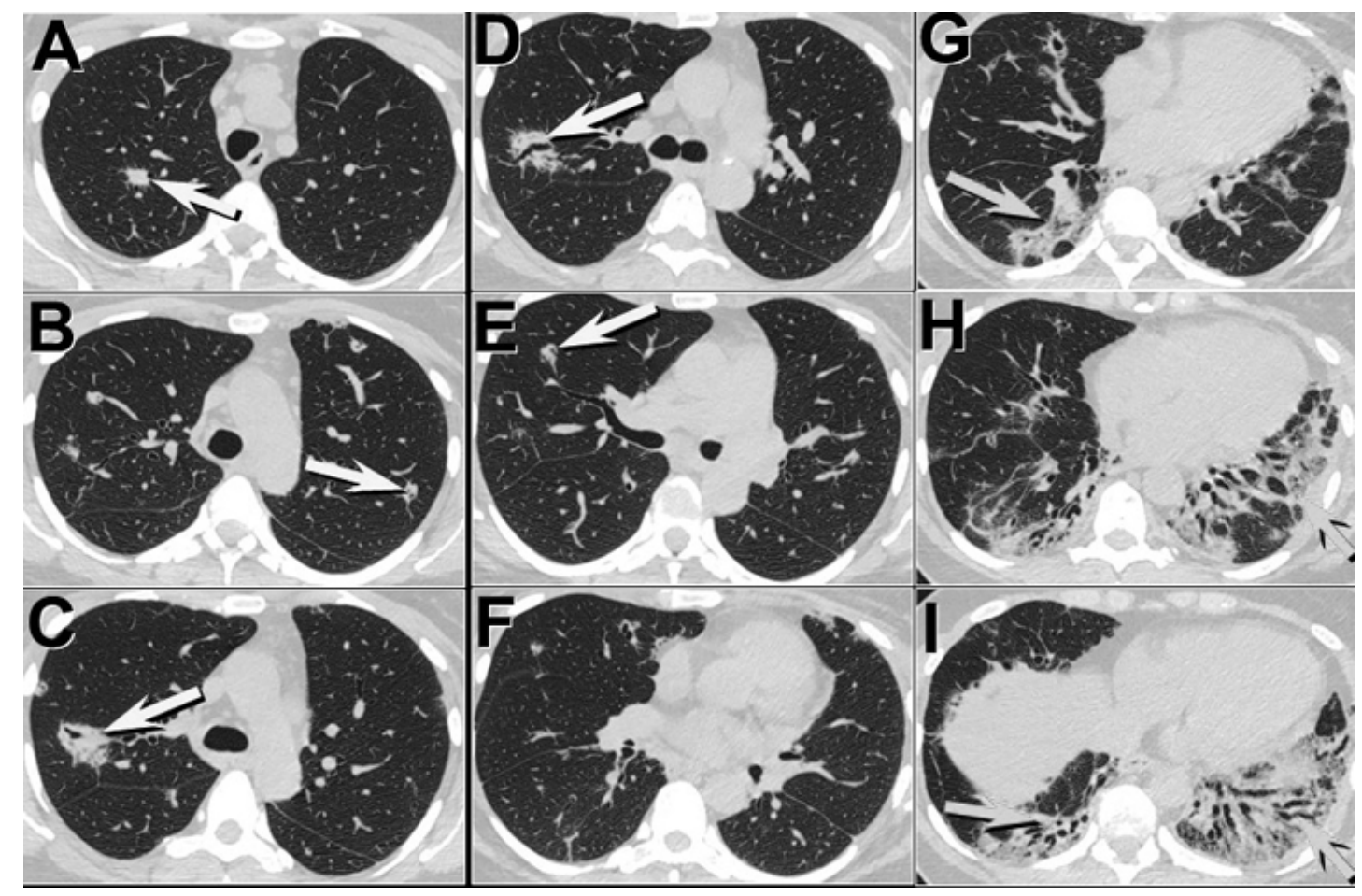

Figure 9. Repeat thoracic CT scan several months later. 
Which of the following represents the most appropriate next step for the management of this patient?

1. Bronchoscopy with bronchoalveolar lavage and transbronchial biopsy

2. Percutaneous fine needle aspiration / core biopsy

3. Repeat thoracoscopic lung biopsy

4. All of the above

5. None of the above 


\section{Correct! \\ 2. Percutaneous fine needle aspiration / core biopsy}

While it may seem that all three tissue sampling procedures may again be equally appropriate, and the risk / benefit ratio is probably similar for all, percutaneous transthoracic fine needle aspiration / core biopsy is probably the best choice at this point. While surgical lung biopsy provides the largest tissue sample, and this benefit is often equated with optimal diagnostic accuracy, it generally provides tissue from the more peripheral aspects of the lung. Therefore, if the areas requiring targeting are located in deeper ling tissue, a surgical lung biopsy may not offer the best opportunity for diagnosis. In this patient, and adequate pulmonary parenchymal tissue sample was already obtained from the let lower lobe, and significant histopathologic abnormalities were seen, but none adequately explained the patient's complaints and progressive scan abnormalities. On the thoracic CTs shown previously, several nodular lesions, most notably in the right upper lobe, have shown progression, and would provide good targets for tissue sampling. Bronchoscopy with bronchoalveolar lavage and transbronchial biopsy has the potential to provide a diagnosis in this patient, particularly given the bronchiolocentric nature of the dominant right upper lobe lesion(see panels $\mathrm{C}$ and D, Figure 9), but percutaneous fine needle aspiration / core biopsy can provide a larger amount of tissue sample for diagnosis than a transbronchial biopsy and allows direct visualization of the lesion to be targeted- these advantages of percutaneous fine needle aspiration / core biopsy make the best choice for a tissue diagnosis for this patient.

Further clinical course: The patient subsequently percutaneous fine needle aspiration / core biopsy (Figure 10), with the material retrieved showing B cell lymphocytes staining positive for CD 20, CD 3, CD 43, and CD 5, and absent staining for cyclin D1, all consistent with small lymphocytic lymphoma.

Diagnosis: Small lymphocytic lymphoma non-Hodgkin lymphoma, recurrent in the lung

\section{References}

1. Albores J, Fishbein MC, Wang T. A 57-year-old woman with persistent cough and pulmonary nodules. Chest 2014;145(5):1162-5. [CrossRef] [PubMed]

2. Do KH, Lee JS, Seo JB, Song JW, Chung MJ, Heo JN, Song KS, Lim TH. Pulmonary parenchymal involvement of low-grade lymphoproliferative disorders. J Comput Assist Tomogr 2005;29(6):825-30. [CrossRef] [PubMed]

3. Hare SS, Souza CA, Bain G, Seely JM, Gomes MM, Quigley M. The radiological spectrum of pulmonary lymphoproliferative disease. Br J Radiol 2012; 85(1015):84864. [CrossRef] [PubMed]

4. Hwang GL, Leung AN, Zinck SE, Berry GJ. Recurrent lymphoma of the lung: computed tomography appearance. J Comput Assist Tomogr 2005;29(2):228-30. [CrossRef] [PubMed]

5. Michael CW, Richardson PH, Boudreaux CW. Pulmonary lymphoma of the mucosaassociated lymphoid tissue type: Report of a case with cytological, histological, 
immunophenotypical correlation, and review of the literature. Ann Diagn Pathol 2005; 9(3):148-52. [CrossRef] [PubMed] 